Meta

Journal des tradlucteurs

Translators' Journal

\title{
La place du syntagme dans le dictionnaire de langue
}

\section{Jean-Claude Boulanger}

Volume 34, numéro 3, septembre 1989

1. Actes du Colloque Les terminologies spécialisées : Approches quantitative et logico-sémantique et 2 . Actes du Colloque Terminologie et Industries de la langue

URI : https://id.erudit.org/iderudit/002111ar

DOI : https://doi.org/10.7202/002111ar

Aller au sommaire du numéro

Éditeur(s)

Les Presses de l'Université de Montréal

ISSN

0026-0452 (imprimé)

1492-1421 (numérique)

Découvrir la revue

Citer cet article

Boulanger, J.-C. (1989). La place du syntagme dans le dictionnaire de langue. Meta, 34(3), 516-528. https://doi.org/10.7202/002111ar d'utilisation que vous pouvez consulter en ligne.

https://apropos.erudit.org/fr/usagers/politique-dutilisation/ 


\section{LA PLACE DU SYNTAGME DANS LE DICTIONNAIRE DE LANGUE}

JEAN-Claude Boulanger Université Laval, Québec, Canada

... a term should be as short as possible and not longer than necessary ...

Sager, 1979, p. 52)

\section{INTRODUCTION}

L'objet de cette communication consiste à examiner la place du syntagme, et plus spécifiquement du syntagme terminologique (ST), dans les dictionnaires de langue ou mieux dans les dictionnaires généraux monolingues (DGM). Le syntagme sera ici défini comme étant «un groupe de mots séparés par des blancs et qui sont syntaxiquement liés tout en identifiant une notion unique dans un domaine déterminé du savoir» (Boulanger, 1988a: 2). Le syntagme est donc une suite de signifiants discontinus empruntant la physionomie d'une séquence syntaxique à combinaisons variables suivant les groupes de mots et à signifié unique et constant. La dénomination syntagme recouvre les synonymes proposés par d'autres linguistes: c'est la lexie complexe de Pottier, la synapsie de Benveniste, le synthème de Martinet, l'unité phraséologique de Dubois, l'unité syntagmatique de Guilbert, etc. (Kocourek, 1982 : 117; Boulanger/Nakos, 1988). La forme composée apparaît aussi fréquemment dans la littérature scientifique européenne. Ce dernier terme n'est cependant que partiellement synonyme de syntagme. L'un comme l'autre relèvent par ailleurs de la polysémie; ce qui ne réjouit guère les terminologues préoccupés par l'équation : un terme $\leftrightarrow$ un concept. Dans la suite de l'exposé, j'utiliserai le terme syntagme et quelques autres variantes comme terme complexe, forme complexe, etc.

La langue, dans ses dimensions générale et spécialisée, propose aux locuteurs des masses d'unités complexes qu'il faut mémoriser et maîtriser à l'égal des formes simples, des locutions, des proverbes et des autres constructions qui font appel à des séquences de mots disjoints; point de vue, bon vivant, accident de parcours, violon d'Ingres sont aussi sémantiquement monolithiques que tourner au vinaigre, à visage découvert, de vieille souche, jamais de la vie ou Quand le diable devient vieux, il se fait ermite («il est facile de renoncer au plaisir quand on ne peut plus le goûter» $(\mathrm{PR})$. Une proportion considérable de mots complexes pénètrent dans les DGM. Alain Rey estime qu'un répertoire de 80000 entrées, comme le GRLF, abrite quelque 500000 ST dans le corps des articles (Rey, 1985a: XXIV, n. 6). L'évaluation toute intuitive du lexicographe robertien n'inclut pas les syntagmes qui appartiennent à la langue générale. Leur nombre est sans nul doute fort élevé.

Comme j'ai déjà abordé ailleurs la question du statut du syntagme dans la macrostructure des DGM (voir Boulanger, 1988a), la présente incursion balisera la microstructure, c'est-à-dire le «programme systématique de présentation et de traitement des unités» (Rey, 1977: V-31). Avant d'entrer dans le vif du sujet, il convient de réanimer deux idées.

1. Une recherche rapide et partielle au sujet des différentes catégories d'entrées dans cinq dictionnaires de langue récents montre que le syntagme occupe environ $1 \%$ de la nomenclature totale (Boulanger, 1988a: tableaux 3 et 4). «Ce type de formations composées [les syntagmes] n'était pas reconnu dans la lexicographie traditionnelle; celles-ci 
étaient recensées à l'intérieur des articles comme séquences phraséologiques servant d'exemples à l'appui du classement sémantique. La linguistique moderne a dégagé la spécificité de ces formations sans parvenir encore à bouleverser la présentation des dictionnaires» (Guilbert, 1971: LXVII). À titre d'exemple, le mot homme, prolifique sur le plan syntagmatique, n'a pas droit à une seule entrée sous l'une ou l'autre de ses apparences complexes dans le PLI89 et le MR88. Les articles s'étendent sur deux-tiers de colonne; ils recèlent respectivement douze et 23 syntagmes construits à l'aide du mot homme dans le rôle de la base ou de l'expansion. Au total, on recense 29 formes différentes (les formes communes portent l'astérique dans le tableau 1). Le signe moins placé entre crochets [-] signale que le syntagme est répertorié dans le dictionnaire sous l'autre composante. Trois unités du GRLF se retrouvent ailleurs (grand homme, homme de main et homme de troupe) tandis que huit unités du PLI subissent le même sort (homme de bien, homme de génie, homme de la rue, homme de lettres, homme de peine, jeune homme, vieil homme (sous vieux) et voix d' homme). Aucune des formes en question n'est commune aux deux dictionnaires. Afin de compléter le carrousel, il resterait à vérifier les syntagmes qui ont les honneurs des articles consacrés à chaque élément autonome qui les compose. Cette recherche est en cours.

Tableau 1: Le syntagme homme dans le PLI89 et le MR88

\begin{tabular}{|c|c|c|c|}
\hline homme & GRLF & PLI & * \\
\hline $\begin{array}{l}\sim \text { à femmes } \\
\sim \text { d'action } \\
\sim \text { d'affaires } \\
\sim \text { de bien } \\
\sim \text { de Cro-Magnon } \\
\sim \text { de génie } \\
\sim \text { de la rue } \\
\sim \text { de lettres } \\
\sim \text { de loi } \\
\sim \text { de main } \\
\sim \text { de peine } \\
\sim \text { de science } \\
\sim \text { d'État } \\
\sim \text { de troupe } \\
\sim \text { du jour } \\
\sim \text { du monde } \\
\sim \text { du peuple } \\
\sim \text { du rang } \\
\sim \text { marié }\end{array}$ & $\begin{array}{l}+ \\
+ \\
+ \\
+ \\
- \\
+ \\
+ \\
+ \\
+ \\
{[-]} \\
+ \\
+ \\
+ \\
{[-]} \\
+ \\
+ \\
+ \\
- \\
+\end{array}$ & $\begin{array}{l}- \\
+ \\
+ \\
{[-]} \\
+ \\
{[-]} \\
{[-]} \\
{[-]} \\
+ \\
+ \\
{[-]} \\
- \\
+ \\
+ \\
- \\
+ \\
- \\
+ \\
-\end{array}$ & $\begin{array}{l}* \\
*\end{array}$ \\
\hline $\begin{array}{l}\text { âge d' } ~ \\
\text { droits de l' } \\
\text { Fils de l' } \\
\text { grand } \sim \\
\text { jeune } \sim \\
\text { parole d' } ~ \\
\text { premier } \sim \\
\text { vêtement d' } ~ \\
\text { vieil } \sim \\
\text { voix d' } ~\end{array}$ & $\begin{array}{l}+ \\
+ \\
+ \\
{[-]} \\
+ \\
+ \\
- \\
+ \\
+ \\
+\end{array}$ & $\begin{array}{c}- \\
- \\
+ \\
+ \\
{[-]} \\
- \\
+ \\
- \\
{[-]} \\
{[-]}\end{array}$ & $*$ \\
\hline 29 & 23 & 12 & 6 \\
\hline
\end{tabular}


2. Le syntagme constitue la caractéristique dominante en terminologie. Les statistiques compilées à propos de différents corpus montrent que le pourcentage moyen de ST pivote autour de $80 \%$ de la masse des termes répertoriés dans une sphère de la connaissance (voir Boulanger, 1988a, tableaux 1 et 2). Aucune statistique n'est disponible en ce qui concerne la proportion de syntagmes en langue générale.

\section{AUTOPSIE DU SYNTAGME}

Un volume assez considérable de formes complexes passe dans les DGM. Le lexicographe doit donc définir des modalités d'intégration et de traitement qui puissent correspondre à son programme dictionnairique et satisfaire le public consulteur. En lexicographie française, la tradition veut qu'on n'accorde pas spontanément le statut d'entrée à tous les syntagmes, généraux comme spécialisés. Ces formes libres unies par un contenu sémantique et une liaison syntaxique qui caractérisent leur fusion n'ont pas fait l'objet de «critères de délimitation entre celles qui accèdent au statut d'entrée et celles qui en sont écartées» (Guilbert/Lagane/Niobey, 1971: III). S'ils existent, ces critères sont pour le moins imprécis ou ils ne sont jamais évoqués dans les discours prélexicographiques. L'un des critères avancés est la prédominance de la forme simple ou continue du mot sur le sens. «La définition des unités composées, en effet, n'est pas fixée d'une manière indiscutable. La lexicologie traditionnelle reconnaît comme mots composés ceux dont les termes constituants peuvent être soit soudés (portefeuille), soit unis par la marque graphique du trait d'union; ils sont recensés à leur ordre alphabétique comme unités indépendantes. Les unités formées de plusieurs éléments lexicaux autonomes [...] sont recensées sous l'adresse du premier terme constituant. À peine une évolution récente, dont ce dictionnaire [le GLLF] s'est fait l'écho, amène-t-elle à considérer chemin de fer et pomme de terre comme des entrées séparées» (Guilbert, 1971 : IX).

Ainsi si l'on convient qu'un grand nombre de syntagmes sont élaborés à l'aide d'unités générales autonomes en langue, c'est-à-dire qu'elles fonctionnent aussi comme des mots individualisés déjà disponibles dans le fonds commun du lexique, ou virtuelles en raison de leur caractère néologique, et, le plus souvent polysémique du point de vue lexicographique, on considérera leur association comme normale et réalisable par le locuteur au gré de ses besoins discursifs et selon les mêmes mécanismes de formation auxquels il recourt pour créer des unités simples. Cette conception entraîne que chaque segment du dérivé syntagmatique peut être répertorié à sa place alphabétique dans le dictionnaire de langue. La refragmentation du terme complexe en ses parties constituantes élimine le plus souvent les joncteurs syntaxiques qui jouent pourtant un rôle fondamental en tant que courroie de transmission lors de l'établissement du sens du syntagme. Cette fonction est aussi primordiale que le contexte situationnel extralinguistique. À titre d'exemple, dans le Vocabulaire des papiers et cartons (Côte, 1983), il y a 108 ST dérivés de la base carton. Les expansions utilisent un total de 112 constituants différents auxquels s'additionnent 5 joncteurs produisant 63 occurrences syntagmatiques (à $[a u]$ avec 35 apparitions, de [de la, de l'] avec 17 apparitions, pour, avec neuf apparitions, sur et une avec une attestation chacun). Par expansion il faut entendre aussi bien une forme libre comme jute, contrecollé ou bloc-notes qu'un morphème lié comme mi-, mono(-) ou simili(-) qui ont été détachés de leur élément lexical dans le corpus étudié ici. Le GRLF enregistre la totalité des formes en entrées indépendantes tandis que le PLI en retient 99. Aucun mot n'est rejeté par les deux dictionnaires. La liste alphabétique de toutes les unités et des éléments de formation est dressée dans l'annexe. Elles sont accompagnées de la catégorisation lexicale (67 noms, 39 adjectifs, deux verbes, quatre préfixes) et de l'indicatif de fréquence. Les joncteurs n'apparaissent pas dans la liste tandis que les expansions elles-mêmes syntagmatiques ont été fragmentées et comptées comme des mots simples 
(ex. : boitte d'allumettes > boîte et allumette ; carte postale > carte et postal). Les composés soudés ou unis par le trait d'union sont restés intacts (ex. : contre-collé, bloc-notes); Les dérivés à préfixes liés ont été décomposés (ex.: monocouche > mono-, couche; simili-chêne $>$ simili-, chêne). Enfin, la forme canonique a été rétablie (ex. cartes $>$ carte; perforée $>$ perforé). La réunion des formes simples en agrégat syntagmatique n'est cependant pas toujours prévisible et elle pose des problèmes au plan sémantique, difficultés que les consulteurs de dictionnaires souhaitent justement voir résolues dans leur outil de référence préféré. Si carton blanc, carton à calendrier et carton ondulé se décodent aisément à partir des sens premiers des éléments, il n'en va pas de même de tous les assemblages, tant s'en faut. Que déduire de carton fossile («Carton composé de fibres d'amiante uniquement ou de fibres d'amiante mélangées avec des liants ou charges, utilisé comme isolant thermique et électrique en raison de son haut degré d'incombustibilité» (Côte, 1983: 13), de carton à crachoir ( «Carton imperméable, le plus souvent ciré, servant à fabriquer les gobelets que l'on place dans les petits crachoirs utilisés dans les hôpitaux et que l'on jette après usage» (Côte, 1983: 54)) et de carton diélectrique («Carton dur et dense servant d'isolant électrique» (Côte, 1983: 29)? Les sens de ces syntagmes sont loin de se laisser deviner de la simple projection faite à l'aide des informations sémantiques recueillies dans les DGM pour chacun des exemples cités.

\section{DE LA MACROSTRUCTURE À LA MICROSTRUCTURE}

S'il est souhaitable d'améliorer le statut du syntagme dans les DGM, c'est sûrement au sein de la microstructure que les hésitations sont les plus perceptibles et que les règles sont les plus difficiles à structurer.

Une spectroscopie de l'article de dictionnaire révèle rapidement que le syntagme peut loger dans presque toutes les rubriques microstructurales, à l'exception bien entendu de la prononciation et de la catégorisation lexico-grammaticale, quoiqu'on y trouve des traces indirectes parfois (voir MR: grand appartement: [gr tapart $\mathrm{m}$ ], sous grand et GRLF : 3.Pandore (boîte de) : loc. nominale). La sous-entrée, l'exemple construit ou littéraire et la définition sont les rubriques qui accueillent le plus fréquemment les contingents lexicaux sémantiquement figés.

À ce stade, il est également bon de rappeler que le syntagme n'est pas l'unique groupe de mots ayant un sens personnalisé, tout en étant syntaxiquement liés, qui figure dans les articles: les locutions, les proverbes, les phraséolo- gismes, les constructions diverses sont également catalogués. Les deux premières catégories d'expressions sont souvent labellisées (voir l'origine de ce mot dans Boulanger, 1988b) à l'aide des abréviations loc. et prov., sort enviable qui est loin d'être réservé aux unités complexes. Voir par exemple l'article chèque dans le GRLF: Loc. fig. Donner un chèque en blanc à qqn.

Afin d'illustrer l'importance et les rôles du syntagme dans les DGM, j'ai choisi d'examiner l'article chèque dans le GRLF. Le texte occupe les deux-tiers d'une colonne. En ne comptabilisant pas les formules phraséologiques comme émettre un chèque et faire porter un chèque au crédit de son compte ainsi que la locution citée précédemment, on repère 32 dérivés complexes dûment «coalescencés» sémantiquement. (L'évaluation de leur degré de figement sémantique n'est redevable qu'à moi seul.) Parmi les 32 complexes lexicaux, 26 sont construits à l'aide du terme chèque, qui sert de base à vingt occasions et d'expansion déterminative à six reprises. Les six syntagmes restants font appel à d'autres termes que chèque (voir le tableau 2).

Les syntagmes ont également été classés dans une grille de modèles de base emprunté à Louis Guilbert (voir Guilbert, 1970). Un septième type, assimilé par Guilbert aux structures à bases nominales, a été ici considéré. Les raisons évoquées par le regretté linguiste pour fusionner les modèles à base verbale aux schémas nominaux ne résistent 
pas toutes à l'analyse, particulièrement dans les technolectes. Ils ne peuvent pas tous être nominalisés à partir de la forme passive du verbe qui fournit un substantif déverbal; ainsi la phrase ce qui fait que les fonds sont appelés se transforme en appel de fonds alors que la phrase ce qui fait que le versement est appelé, qui est aussi nominalisable, n'est pas nominalisée en *appel de versement dans le domaine bancaire; le groupe appeler un versement est privilégié par les utilisateurs. Il en va de même de ce qui fait que le rappel est sonné qui ne se transforme pas puisque dans ce cas, il n'existe pas de déverbal de sonner, ni de substitut qui ferait référence à «un radical différent de celui du verbe de base» (Guilbert, 1971: LXVII ; voir aussi l'analyse transformationnelle des locutions verbales avancée par l'auteur à la p. LXVIII).

La grille illustre la fréquence et le pourcentage pour chaque modèle (voir le tableau 3 ). Le numéro du schéma binaire est le même qui apparaît dans le tableau 2.

Des six modèles binaires guilbertiens, quatre sont représentés dans le minicorpus témoin tandis que deux sont absents. On en trouve cependant de nombreuses attestations ailleurs dans le GRLF : petit déjeuner* pour le modèle 5, permis* de conduire pour le

Tableau 2: Les syntagmes dans l'article chèque du GRLF

\begin{tabular}{|c|c|c|}
\hline syntagme & modèle & structure \\
\hline 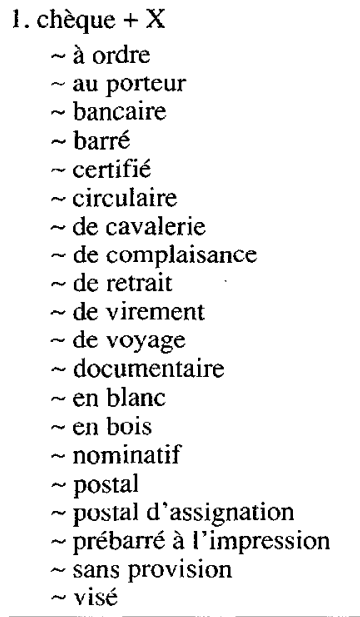 & $\begin{array}{l}1 \\
4 \\
2 \\
2 \\
2 \\
2 \\
1 \\
1 \\
1 \\
1 \\
1 \\
2 \\
1 \\
1 \\
2 \\
2 \\
1 \\
4 \\
1 \\
2\end{array}$ & 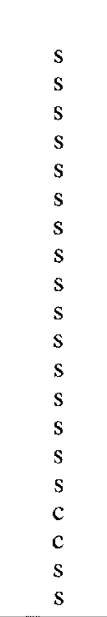 \\
\hline $\begin{array}{l}\text { 2. } \mathrm{X}+\text { chèque }(\mathrm{s}) \\
\text { carnet de } \sim \mathrm{s} \\
\text { formule de } \sim \\
\text { payer par } \sim \\
\text { versement par } \sim\end{array}$ & $\begin{array}{l}1 \\
1 \\
7 \\
1\end{array}$ & $\begin{array}{l}\mathrm{s} \\
\mathrm{s} \\
\mathrm{s} \\
\mathrm{s}\end{array}$ \\
\hline $\begin{array}{l}\text { 3. } X+\text { chèque(s) }+X \\
\text { bureau de chèques postaux } \\
\text { compte chèque postal }\end{array}$ & $\begin{array}{l}1 \\
3\end{array}$ & $\begin{array}{l}\mathrm{c} \\
\mathrm{c}\end{array}$ \\
\hline $\begin{array}{l}\text { 4. autres combinaisons } \\
\text { agent de change } \\
\text { barres parallèles } \\
\text { carte blanche } \\
\text { compte postal } \\
\text { dispositions légales } \\
\text { établissement financier }\end{array}$ & $\begin{array}{l}1 \\
2 \\
2 \\
2 \\
2 \\
2\end{array}$ & $\begin{array}{l}\mathrm{s} \\
\mathrm{s} \\
\mathrm{s} \\
\mathrm{s} \\
\mathrm{s} \\
\mathrm{s}\end{array}$ \\
\hline
\end{tabular}


modèle 6. Enfin, les syntagmes simples (28/32 ou $87,5 \%$ ) l'emportent largement sur les structures plus complexes (4/32 ou 12,5\%), c'est-à-dire des syntagmes eux-mêmes dérivés de constructions bipolaires.

Dans le tableau 2, les lettres [S] et [C] identifient l'agencement primaire ou l'agencement plus développé.

Tableau 3: Typologie des syntagmes : modèles binaires

\begin{tabular}{|l|c|c|}
\hline modèle & nombre & pourcentage \\
\hline 1. nom + joncteur + nom & 15 & $\sim 47 \%$ \\
\hline 2. nom + adjectif & 13 & $\sim 41 \%$ \\
\hline 3. nom $+\emptyset+$ nom & 1 & $\sim 3 \%$ \\
\hline 4. nom + joncteur + prédéterminant + nom & 2 & $\sim 6 \%$ \\
\hline 5. adjectif + nom & 0 & $0 \%$ \\
\hline 6. nom + joncteur + verbe & 0 & $0 \%$ \\
\hline 7. verbe + joncteur + nom & 1 & $\sim 3 \%$ \\
\hline & 32 & $100 \%$ \\
\hline
\end{tabular}

\section{PLACE DU SYNTAGME DANS L'ARTICLE}

Le minicorpus scruté montre que le syntagme peut prendre place dans différentes rubriques et sous-rubriques citées ci-dessous en ordre décroissant d'occurrences observées (voir aussi le tableau 4):

exemple : 1 . exemple construit

1.1 syntagme-exemple (chèque bancaire, payer par chèque);

1.2 segment phrastique (Un carnet de chèques.);

1.3 phrase nominale (Des chèques de voyage en dollars, en yens.);

1.4 contexte terminologique complet non référencé (Les dispositions légales en vigueur ont répandu, depuis 1979 , l'usage des chèques «prébarrés» à l'impression.);

2. citation

2.1 littéraire (chèque en blanc, citation de Roger Garaudy pour le sens figuré);

- définition (établissement financier, agent de change, bureau de chèques postaux);

- réseau analogique ( $\rightarrow$ Donner carte* blanche);

- sous-entrée (chèque postal, chèque de voyage);

- locution (Donner un chèque en blanc à qqn);

- indicatif de région ((Au Canada). Chèque visé).

Un même syntagme peut être retrouvé dans plus d'une rubrique car sa fonction peut changer. Chèque en blanc a été repéré dans un exemple lexicographique qui joue le rôle d'une sous-entrée suivie de sa définition et précédée de la date de première attestation (1953), dans une locution figurée et dans une citation littéraire. Outre ses trois occurrences, ce syntagme constitue à lui seul un sous-article au sein de l'article chèque. Il en va de même pour chèque de voyage pour lequel on fournit la date d'apparition (1953) et l'origine (de l'anglais traveller check). 
Il est parfois fort difficile de distinguer la véritable sous-entrée, repérable grâce à l'information codée que recèle le recours aux petites majuscules (chèque postal), de la sous-entrée caméléon qui emprunte la couleur des italiques, indice habituel de l'exemple (chèque en blanc). En principe, le code lexicographique stipule que les jeux de caractères alternent suivant une procédure rigoureuse qui permet une identification rapide des rubriques et de l'information transmise.

En outre, certains syntagmes-exemples sont prolongés par une définition qui relaie l'équivalent sémantique complet. Cette définition reprend la base du syntagme comme genre prochain (chèque en blanc: «chèque que le tireur a signé sans indiquer la somme que le tiré devra payer») tandis que d'autres n'énumèrent que les différences spécifiques ou ils appartiennent au type de définition relationnelle (chèque certifié, «sur lequel le tiré certifie que la provision du tireur permet de payer le chèque»; chèque de virement et surtout chèque barré qui est suivi d'un segment définitionnel d'un haut degré technolectal). L'utilisation de la «demi-définition» est un choix méthodologique. Alain Rey l'explique comme étant «une sorte de glose [...] analysant un surplus de sens ajouté à ce qui vient d'être défini» (Rey, 1985a, p. XXXIV), c'est-à-dire à la base chèque.

\begin{tabular}{|l|c|}
\hline rubrique & occurrences \\
\hline exemple & \\
$1 . \quad$ exemple construit & 22 \\
1.1. syntagme-exemple & 1 \\
1.2. segment phrastique & 1 \\
1.3. phrase nominale & 1 \\
1.4. contexte terminologique complet & 1 \\
2. citation & 6 \\
\hline définition & 5 \\
\hline réseau analogique & 2 \\
\hline sous-entrée & 1 \\
\hline locution & 1 \\
\hline indicatif de région & \\
\hline
\end{tabular}

Trois syntagmes voient l'une de leurs composantes affublée d'un astérisque qui renvoie à l'autre élément consigné dans le dictionnaire (chèque en bois*, chèque sans provision*, chèque à ordre*). Sous bois, chèque en bois est défini: «chèque sans provision» et il n'y a pas de renvoi à chèque. Chèque sans provision est défini sous ce dernier terme. Malgré l'astérisque de réciprocité, l'article ordre ne récupère pas le syntagme chèque à ordre.

À l'inverse, des syntagmes non marqués à l'aide d'un astérisque se retrouvent aussi sous l'autre forme indépendante: chèque au porteur est inclus dans l'article porteur avec un renvoi à chèque; chèque bancaire apparait sous bancaire avec l'indication : par opposition à chèque postal mais il n'y a pas de renvoi à chèque; chèque barré est sous 1 . barrer sans renvoi réciproque; chèque de cavalerie est défini sous cavalerie sans renvoi à chèque; chèque de complaisance est défini sous le dernier élément sans renvoi; etc. On 
voit déjà poindre ici la nécessité d'intégrer davantage l'informatique à la démarche de rédaction des articles lexicographiques.

L'astérisque sert aussi à identifier le mot-pivot sous lequel trouver une information (carte* blanche dans la locution donner carte* blanche). Il peut enfin arriver que seul le contexte d'utilisation présente un syntagme dont il n'est pas fait mention ailleurs dans l'article (chèque prébarré à l'impression est révélé dans le contexte terminologique).

Un survol rapide de quelques articles montrent que le syntagme est présent en d'autres lieux de la microstructure:

- dans la rubrique étymologique principale (GRLF: parangon : pierre de touche et pierre à aiguiser) ou secondaire (GRLF: chèque de voyage : traveller check);

- dans un exemple de littérature scripto-orale (GRLF: plat pays illustré par un vers de Jacques Brel: Ce plat pays qui est le mien);

- dans le bloc des renvois en fin d'article (GRLF : chemin : DÉR. et COMP. : chemin de fer);

- dans les remarques grammaticales (PR88 : abri-sous-roche: «On écrit parfois abri sous roche». Cette remarque est confirmée dans le GRLF par un exemple tiré de la littérature documentaire alors que l'entrée comporte des traits d'union; elle est toutefois suivie du commentaire: «avec ou sans traits d'union»;

- dans le réseau notionnel (GRLF : abri, dans la section des renvois consacrée aux types d'abris militaires, la liste inclut le syntagme plaque de blindage);

- dans les commentaires métalinguistiques (GRLF : chien: REM. La nomenclature des races de chiens est plus abondante, elle comprend de nombreux emprunts (notamment des anglicismes) et des syntagmes formés avec chien ou avec le nom d'une race (berger, lévrier...). On classe les chiens en groupes: chiens de berger, chiens de garde et de protection, chiens de trait, terriers, teckels, chiens courants, chiens de chasse et chiens d'arrêts, chiens d'agrément et de compagnie, lévriers. - Les chiens de chasse (au sens large) se divisent en chiens d'arrêt, chiens courants, chiens d'équipage, chiens quêteurs, chiens rapporteur et chiens de terrier).

\section{CONCLUSION : Y A-T-IL UN AVENIR POUR LE SYNTAGME EN LEXICOGRAPHIE ?}

L'insertion du syntagme dans un DGM relève d'une décision à deux paliers:

1. Faut-il considérer le syntagme comme un objet lexicographique? 2. Si oui, prendra-t-il place dans la macrostructure ou dans la seule microstructure et quel traitement recevra-til? Dans l'article, il aura un statut de dépendance à l'égard d'un mot-pivot qui servira de fil d'Ariane pour accéder au complexe. Il pourra alors apparaître dans plusieurs rubriques. De nombreux syntagmes sont cependant traités sous chacune de leurs composantes lexicales, ce qui est loin d'être économique mais semble rendre pour le moment de précieux services aux consulteurs.

Indéniablement, le syntagme est un objet lexicographique. On le trouve en macroet en microstructure. «Lorsque le syntagme est lexicalisé, le lexicographe se doit de le définir comme il définit l'entrée. Sans cette définition son statut reste quelconque» (ReyDebove, 1971, p. 303). Plus encore, le processus de lexicalisation ne peut pas être dissocié de la cohésion sémantique, de la référence au contenu de la réalia ou de la notion dont les propriétés se définissent par opposition aux traits propres à d'autres unités. La présence de l'expansion déterminative est obligatoire et nécessaire pour exprimer les caractères sémantiques qui circonscrivent complètement l'objet et le hiérarchise par rapports à d'autres qui appartiennent à la même sphère notionnelle (voir Guilbert, 1971, p. LXIX). Il est clair cependant qu'en lexicographie générale, le réseau notionel dégagé n'a pas le même objectif classificatoire qu'en terminographie. Le DGM décrit le fonctionnement 
des unités de la langue; ce n'est qu'accessoirement qu'il réfère aux classes d'objets de l'univers (voir Rey, 1985b).

De plus, le lexicographe a besoin du syntagme pour élaborer ses énoncés lexicographiques (définition, remarques, interventions diverses, etc.). Le dictionnariste est aussi un écrivain, un manieur de mots qui puise au même réservoir que tous ceux qui écrivent et rédigent. Ainsi dans l'article cheval du MR, on retrace les syntagmes animal de trait, animal de transport, race pure, santé de fer, unité de travail, viande de cheval et course à cheval, tous utilisés pour discourir sur le mot cheval qui est le sujet de l'article. La présence des formations complexes dans les dictionnaires n'est pas une lubie, ni une fantaisie. Elles sont plus que des objets linguistiques à décrire se présentant au lexicographe selon des formules variées. Elles sont elles-mêmes des éléments-clés de l'appareil de description et du discours lexicographiques. Tous les rédacteurs de dictionnaires en conviennent. Mais pour le moment leurs constats s'arrêtent au palier métalinguistique dont les règles sont dictées par la lexicologie traditionnelle. La prise en compte de plus en plus évidente de l'aspect sémantique forcera les lexicographes à franchir la frontière du mot graphique pour octroyer au syntagme la juste place qui lui revient dans les dictionnaires de la langue française, renforcissant ainsi les intelligences avec la terminographie.

\section{IDLL ET ARTISANAT LEXICOGRAPHIQUE}

Les lexicographes façonnent des dictionnaires, c'est-à-dire des instruments du savoir, des produits qui véhiculent la langue et transmettent la vision du monde que se forge chaque communauté linguistique. Les dictionnaires renferment donc en filigrane des mots l'histoire des civilisations, tant dans les dimensions du permis que dans celles de l'interdit (voir Boulanger, 1986). Depuis des siècles, les dictionnaristes œuvrent artisanalement. Longtemps avant la forte poussée expansionniste de l'informatique et de ses outils logiciels, les dictionnaires imprimés constituaient à leur manière une industrie de la langue, de la culture et de la connaissance dont le poids n'était pas négligeable dans l'économie des sociétés européennes. «Terminologie et lexicographie sont des outils de développement de la langue et peuvent, à ce titre, être classées parmi les activités et les produits des industries de la langue» (Auger, 1988 p. 12). Il paraît opportun de tenir compte d'une manière un peu plus affirmée du sens plus «ancien» mais jamais enregistré du syntagme industrie de la langue, à savoir l'introduction des dimensions relatives aux recherches lexicographiques, et même grammaticales, ainsi que leurs produits.

À n'en pas douter, il existe deux visions du concept des «industries de la langue»: la première est une perception informatique qui veut que les IDLL consistent à traiter une langue naturelle à l'aide de l'ordinateur et pour l'ordinateur, que l'intervention de ce dernier soit centrale ou périphérique; la seconde est une perception linguistique qui stipule que les IDLL sont un ensemble d'activités «visant à concevoir, fabriquer et commercialiser des appareils et des logiciels qui manipulent, interprètent et génèrent le langage humain, aussi bien sous sa forme écrite que sous sa forme parlée» (L'Homme, 1988, p. 71). Les produits qui naissent de l'activité industrielle doivent être diffusés et rejoindre les publics pour lesquels ils sont conçus.

Dans l'exposé qui a précédé, on a pu montrer que les formes composites (constructions, syntagmes, expressions, locutions, etc.) «que le lexicographe met en cage et en listes» (Rey, 1985a, p. XXXV), jouent un rôle quantitatif et qualitatif prépondérant dans les DGM. Dans plusieurs répertoires, on a entrepris la systématisation des constructions, on a commencé à sortir les mots de leur isolement graphique pour leur donner un statut un peu plus en adéquation avec leur valeur sémantique, notamment en leur ouvrant les portes de la sous-entrée (voir MR: 1 . trait: AU TRAIT, À GRANDS TRAITS, TRAIT DE..., AVOIR TRAIT À). En raison de sa coalescence sémantique, le syntagme général 
ou terminologique réclame la même naturalisation macro- ou microstructurale. Il faudrait le sortir de l'antichambre de l'à-peu-près et le pourvoir de critères rigoureux et constants, appliqués systématiquement et qui en régissent la consignation.

Le renforcement de la présence du syntagme dans les dictionnaires est lié à un aspect des industries de la langue qu'il faut maintenant préciser. La lexicographie générale a tout intérêt à développer des logiciels de découpage des mots qui aillent au-delà de la forme simple pour intégrer des séquences graphiques interrompues. Il est clair que l'un des problèmes les plus difficiles que devront résoudre les logiciels de reconnaissance des mots est sans contredit l'himalaya que représente les assemblages de formations complexes à sens unique. Et lorsque la sémantique se mêle de la partie, on voit poindre le nez de l'intelligence artificielle. Le défi des lexicographes est ici identique à ceux qui ont déjà été identifiés pour d'autres zones de recherche dans le secteur des IDLL. En tant qu'activité linguistique qui doit se pencher sur les comportements morphologiques, syntaxiques et sémantiques des plurimots et en tant que produit commercial, le dictionnaire de langue nécessite un apport informatique en amont et en aval de son élaboration. Perçus sous cet angle, la lexicographie et le dictionnaire sont aussi des créatures des IDLL. Personne ne le contestera lorsque chacun pourra «ouvrir» son Robert ou son Larousse en poussant quelques boutons de la télécommande ou du clavier de son minitel ou en faisant tourner le vidéodisque de son Dictionnaire thématique visuel. Ces gestes seront bientôt quotidiens et naturels. Ils n'appartiennent déjà plus au domaine de l'imaginaire ni à celui de la linguistique-fiction. Ils sont déjà entrés dans l'histoire de la lexicographie et de la linguismatique. Encore faut-il que chaque question trouve sa réponse! C'est à mon sens I'un des grands défis immédiats de la lexicographie.

\section{BIBLIOGRAPHIE}

\section{Linguistique}

AUGER, Pierre (1988) : «Le travail du terminologue amélioré et simplifié», Circuit, n²2, septembre, pp. 12-13.

BOULANGER, Jean-Claude (1986): Aspects de l'interdiction dans la lexicographie française contemporaine, coll. «Lexicographica», Series Maior, no 13, Tübingen, Max Niemeyer Verlag, IX + $166 \mathrm{p}$.

BOULANGER, Jean-Claude (1988a): «Le statut du syntagme dans les dictionnaires généraux monolingues», (dans ce numéro).

BOULANGER, Jean-Claude (1988b) : «Remarques sur l'aménagement du statut du français en informatique», Annie Bourret et Marie-Claude L'Homme (éd.), Les industries de la langue : au confluent de la linguistique et de l'informatique, sous la direction de Pierre Auger, avec la collaboration de Carole Verreault], coll. «K», $n^{\circ}$ 9, Québec, Centre international de recherche sur le bilinguisme, Université Laval, pp. 61-19.

BOULANGER, Jean-Claude) et Dorothy Nakos (1988) : «Le syntagme terminologique» : Bibliographie sélective et analytique (1960-1988), coll. $\ll \mathrm{K} », \mathrm{n}^{\circ} 7$, Québec, Centre international de recherche sur le bilinguisme, Université Laval, $81 \mathrm{p}$.

GUILBERT, Louis (1970) : «La dérivation syntagmatique dans les vocabulaires scientifiques et techniques, dans Les langues de spécialité (Analyse linguistique et recherche pédagogique)», Actes du stage de SaintCloud, 23-30 novembre 1967, Publication du Conseil de l'Europe, Strasbourg, Aidela, p. 116-125.

GUILBERT, Louis (1971): «Fondements lexicologiques du dictionnaire. De la formation des unités lexicales», Grand Larousse de la langue française, Paris, Librairie Larousse, T. 1, pp. IX-LXXXI.

GUILBERT, Louis, René Lagane et Georges Niobey (1971): «Préface. Un nouveau dictionnaire de la langue française», Grand Larousse de la langue française, Paris, Librairie Larousse, T. I, pp. I-V.

KOCOUREK, Rostislav (1982): La langue française de la technique et de la science, Wiesbaden, Oscar Brandstetter, $262 \mathrm{p}$.

L'HOMME, Marie-Claude (1988): [sous la direction de Pierre Auger, Jean-Claude Boulanger et Conrad Ouellon], «Origine et développement des industries de la langue», coll. «K», ${ }^{\circ} 8$, Québec, Centre international de recherche sur le bilinguisme, Université Laval, $\mathrm{V}+141 \mathrm{p}$.

REY, (Alain (1977) : «La terminologie : réflexions sur une pratique et sur sa théorie», Terminologies 76, Actes du Colloque international, Paris-La Défense, 15-18 juin 1976, Paris, La Maison du dictionnaire, pp. V-14-V-40.

REY, Alain (1985a) : «Préface», Le Grand Robert de la langue française, Dictionnaire alphabétique et analogique de la langue française, $2^{e}$ édition entièrement revue et augmentée par A. Rey, Paris, Dictionnaires Le Robert, pp. XVII-XLII. 
REY, Alain (1985b) : «La terminologie dans un dictionnaire général de la langue française : le Grand Robert», TermNet News, $\mathrm{n}^{\circ} 14$, pp. 5-7.

REY-DEBOVE, Josette (1971): Étude linguistique et sémiotique des dictionnaires français contemporains, coll. «Approaches to Semiotics», $\mathrm{n}^{\circ} 13$, The Hague/Paris, Mouton, $331 \mathrm{p}$.

SAGER, Juan Carlos (1979) : «Commentary of Pierre Auger's Paper, «La syntagmatique terminologique, typologie des syntagmes et limite des modèles en structure complexe»», Table ronde sur les problèmes du découpage du terme, Montréal, 26 août 1978, Ve Congrès de l'Association internationale de linguistique appliquée (AILA), 20 au 26 août 1978, Montréal, Office de la langue française, Éditeur officiel du Québec, novembre, pp. 37-52.

Diçtionnaires

CORBEIL, Jean-Claude (1986) : Dictionnaire thématique visuel, Montréal, Les Éditions Québec/Amérique inc. 800 p. [DTV]

CÔTE, Normand (1983): Vocabulaire des papiers et des cartons (anglais-français), coll. «Cahiers de l'Office de la langue française», Québec, Gouvernement du Québec, 79 p.

Grand Larousse de la langue française (1971-1978): [sous la direction de L. Guilbert, R. Lagane, G. Niobey, avec le concours de H. Bonnard, L. Casati, A. Lerond], Paris, Librairie Larousse, 7 vol., CXXVIII +6730 p. [GLLF]

Le Micro-Robert. Langue française plus noms propres, chronologie, cartes (1988), Rédaction dirigée par Alain Rey, Paris, Dictionnaires Le Robert, XXVII + 1992 p. [MR]

Petit Larousse illustré $(1989,1988)$, Paris, Librairie Larousse, 1680 p. + Atlas. [PLI]

ROBERT, Paul (1985) : Le Grand Robert de la langue française. Dictionnaire alphabétique et analogique de la langue française, $2^{\mathrm{e}}$ édition entièrement revue et augmentée par A. Rey, Paris, Dictionnaires Le Robert, 9 vol., LVIII p. + p.v. [GRLF]

ROBERT, Paul (1988): Le Petit Robert 1. Dictionnaire alphabétique et analogique de la langue française, Nouvelle édition revue, corrigée et mise à jour, Rédaction dirigée par Alain Rey et Josette Rey-Debove, Paris, S.N.L. - Dictionnaires Le Robert, XXXI + 2175 p. [PR]

\section{ANNEXES} 1983)

Liste alphabétique des constituants des syntagmes construits avec la base carton (voir Côte,

\begin{tabular}{|l|c|c|c|c|c|}
\hline mot & $\begin{array}{c}\text { catégorie } \\
\text { lexicale }\end{array}$ & $\begin{array}{c}\text { occur- } \\
\text { rences }\end{array}$ & GRLF & PLI & $\begin{array}{c}\text { commun } \\
\text { aux deux } \\
\text { dict. }\end{array}$ \\
\hline accumulateur & nom & 1 & + & + & $*$ \\
affiche & nom & 2 & + & + & $*$ \\
albâtre & nom & 1 & + & + & $*$ \\
allumette & nom & 2 & + & + & $*$ \\
amiante & nom & 1 & + & + & $*$ \\
armé & adj. & 1 & + & + & $*$ \\
billet & nom & 1 & + & + & $*$ \\
bitumé & adj. & 1 & + & - & $\emptyset$ \\
blanc & adj. & 1 & + & + & $*$ \\
bloc-notes & nom & 1 & + & + & $*$ \\
bois & nom & 1 & + & + & $*$ \\
boîte & nom & 1 & + & + & $*$ \\
brillant & adj. & 1 & + & + & $*$ \\
bristol & nom & 4 & + & + & $*$ \\
brun & adj. & 1 & + & + & $*$ \\
brut & adj. & 1 & + & + & $*$ \\
buvard & nom & 1 & + & + & $*$ \\
cadre & nom & 1 & + & + & $*$ \\
calendrier & nom & 1 & + & + & $*$ \\
cannelure & nom & 3 & + & + & $*$ \\
\hline
\end{tabular}




\begin{tabular}{|c|c|c|c|c|c|}
\hline mot & $\begin{array}{c}\text { catégorie } \\
\text { lexicale }\end{array}$ & $\begin{array}{l}\text { occur- } \\
\text { rences }\end{array}$ & GRLF & PLI & $\begin{array}{c}\text { commun } \\
\text { aux deux } \\
\text { dict. }\end{array}$ \\
\hline carrosserie & nom & 1 & + & + & $*$ \\
\hline carte & nom & 3 & + & + & * \\
\hline chaussure & nom & 2 & + & + & * \\
\hline chemin & nom & 1 & + & + & $*$ \\
\hline chemise & nom & 1 & + & + & $*$ \\
\hline chêne & nom & 1 & + & + & $*$ \\
\hline chimique & adj. & 1 & + & + & $*$ \\
\hline chiné & adj. & 1 & + & + & * \\
\hline cible & nom & 1 & + & + & * \\
\hline combiné & adj. & 2 & + & + & $*$ \\
\hline comprimé & adj. & 1 & + & + & $*$ \\
\hline construction & nom & 1 & + & + & $*$ \\
\hline contrecollé & adj. & 4 & + & + & $*$ \\
\hline contrefort & nom & 1 & + & + & $*$ \\
\hline couche & nom & 4 & + & + & * \\
\hline couché & adj. & 4 & + & + & $*$ \\
\hline crachoir & nom & 1 & + & + & $*$ \\
\hline croquis & nom & 1 & + & + & * \\
\hline cuir & nom & 2 & + & + & * \\
\hline dessin & nom & 1 & + & + & $*$ \\
\hline diélectrique & adj. & 1 & + & + & $*$ \\
\hline & nom & 1 & + & + & $*$ \\
\hline double & adj. & 4 & + & + & $*$ \\
\hline doublé & adj. & 1 & + & + & $*$ \\
\hline doublure & nom & 3 & + & + & $*$ \\
\hline duplex & nom & 1 & + & + & $*$ \\
\hline dur & adj. & 1 & + & + & $*$ \\
\hline écru & adj. & 1 & + & + & $*$ \\
\hline emboutissage & nom & 1 & + & + & $*$ \\
\hline entoilé & adj. & 1 & + & - & $\varnothing$ \\
\hline fabriqué & adj. & 1 & + & - & $\emptyset$ \\
\hline face & nom & 4 & + & + & * \\
\hline fer & nom & 1 & + & + & * \\
\hline fibre & nom & 1 & + & + & * \\
\hline fiche & nom & 1 & + & + & $*$ \\
\hline forme & nom & 1 & + & + & * \\
\hline fossile & nom & 1 & + & + & * \\
\hline gabarit & nom & 1 & + & + & $*$ \\
\hline gaufré & adj. & 1 & + & - & $\emptyset$ \\
\hline glacis & nom & 1 & + & + & $*$ \\
\hline goudronné & adj. & 1 & + & - & $\emptyset$ \\
\hline gris & adj. & 7 & + & + & * \\
\hline haut & adj. & 1 & + & + & $*$ \\
\hline huilé & adj. & 1 & + & - & $\emptyset$ \\
\hline imprégné & adj. & 1 & + & - & $\emptyset$ \\
\hline impression & nom & 1 & + & + & $*$ \\
\hline intérieur & adj. & 1 & + & + & $*$ \\
\hline isolant & adj. & 1 & + & + & * \\
\hline ivoire & nom & 2 & + & + & * \\
\hline jacquard & nom & 1 & + & + & * \\
\hline
\end{tabular}




\begin{tabular}{|c|c|c|c|c|c|}
\hline mot & $\begin{array}{l}\text { catégorie } \\
\text { lexicale }\end{array}$ & $\begin{array}{l}\text { occur- } \\
\text { rences }\end{array}$ & GRLF & PLI & $\begin{array}{c}\text { commun } \\
\text { aux deux } \\
\text { dict. }\end{array}$ \\
\hline jouer & verbe & 1 & + & + & $*$ \\
\hline jute & nom & 1 & + & + & $*$ \\
\hline kraft & nom & 2 & + & + & $*$ \\
\hline laqué & adj. & 1 & + & + & $*$ \\
\hline malle & nom & 1 & + & + & * \\
\hline manille & nom & 1 & + & + & $*$ \\
\hline maroquinerie & nom & 1 & + & + & $*$ \\
\hline massicot & nom & 1 & + & + & $*$ \\
\hline matrice & nom & 1 & + & + & $*$ \\
\hline mécanique & adj. & 2 & + & + & $*$ \\
\hline $\mathrm{mi-}$ & préf. & 1 & + & + & $*$ \\
\hline mono- & préf. & 1 & + & - & $\emptyset$ \\
\hline mou & adj. & 1 & + & + & $*$ \\
\hline moulage & nom & 1 & + & + & $*$ \\
\hline multi- & préf. & 1 & + & - & $\emptyset$ \\
\hline ondulé & adj. & 8 & + & + & $*$ \\
\hline onduler & verbe & 1 & + & + & $*$ \\
\hline paille & nom & 2 & + & + & $*$ \\
\hline pâte & nom & 4 & + & + & $*$ \\
\hline patron & nom & 1 & + & + & $*$ \\
\hline perforé & adj. & 1 & + & - & $\emptyset$ \\
\hline plastifié & adj. & 1 & + & - & $\emptyset$ \\
\hline pliage & nom & 1 & + & + & $*$ \\
\hline pochoir & nom & 1 & + & + & $*$ \\
\hline postal & adj. & 1 & + & + & $*$ \\
\hline présentoir & nom & 1 & + & + & $*$ \\
\hline revêtement & nom & 1 & + & + & $*$ \\
\hline rond & adj. & 1 & + & + & $*$ \\
\hline satiné & adj. & 1 & + & + & $*$ \\
\hline semelle & nom & 1 & + & + & * \\
\hline simili- & préf. & 3 & + & - & $\emptyset$ \\
\hline simple & adj. & 2 & + & - & $\emptyset$ \\
\hline soierie & nom & 1 & + & + & * \\
\hline sol & nom & 1 & + & + & $*$ \\
\hline stratifié & adj. & 1 & + & + & * \\
\hline supérieur & adj. & 1 & + & + & * \\
\hline support & nom & 1 & + & + & $*$ \\
\hline talon & nom & 1 & + & + & $*$ \\
\hline ticket & nom & 1 & + & + & $*$ \\
\hline tourbe & nom & 1 & + & + & $*$ \\
\hline triple & nom & 2 & + & + & * \\
\hline tube & nom & 1 & + & + & $*$ \\
\hline 112 & & 166 & 119 & 99 & 99 \\
\hline
\end{tabular}

